\title{
Risiko Pseudarthrose: OP - ja oder nein?
}

Murray et al. identifizieren in einer retrospektiven Studie Risikofaktoren für die Entwicklung einer Pseudarthrose nach konservativ behandelter Klavikulafraktur: An erster Stelle stehen die Größe der Dislokation der Fraktur und bestehender Nikotinabusus.

Murray IR et al., Risk Factors for Nonunion After Nonoperative Treatment of Displaced Midshaft Fractures of the Clavicle J Bone Joint Surg Am, 2013 Jul 03;13:1153-1158.und Kommentar: Schmidt AH. Will my clavicle heal? J. Bone Joint Surg Am. 2013;95:e93

\section{Einleitung \\ $\nabla$}

Bei 5-20\% der Patienten mit konservativ behandelten Klavikulaschaftfrakturen treten Pseudarthrosen auf. Vor allem dislozierte Frakturen zeigen deutlich höhere Rate. Bei Frakturen, die bereits primär durch eine Osteosynthese stabilisiert worden sind, tritt deutlich seltener ein „Nichtheilen“ der Frakturenden auf als bei sekundär versorgten Fällen. Auch findet sich bei der primären Stabilisierung oft ein besseres funktionelles Ergebnis. Betrachtet man nur diese Ergebnisse, könnte man postulieren, alle Klavikulaschaftfrakturen osteosynthetisch zu versorgen. Allerdings sprechen oft andere Gründe gegen eine operative Versorgung, sei es der Allgemeinzustand des Patienten, der ein erhöhtes OP-Risiko darstellt oder der Wunsch des Patienten nach konservativer Therapie. Um hier eine bessere Risikoabschätzung zu ermöglichen, wäre eine Risikostratifizierung für die Entwicklung einer Pseudarthrose bei konservativer Behandlung hilfreich.

Die Autoren untersuchten deshalb, welchen Einfluss mögliche Risikofaktoren auf das Ergebnis und die Entwicklung einer Pseudarthrose haben können.

\section{Methodik}

In der retrospektiven Studie, wurden zwischen 1994 und 2007 insgesamt 941 Patienten eingeschlossen. Dabei handelte es sich um 678 Männer und 263 Frauen, die alle über 18 Jahre alt waren. Eingeschlossen wurden dislozierte (d.h. ohne kortikalen Kontakt) Klavikulafrakturen in den mittleren 3/5 der Klavikula, die nicht pri- mär operativ versorgt worden waren und die vollständig dokumentiert wurden. Neben Alter, Geschlecht, Art des Traumas, betroffener Seite und anderer Erkrankungen, wurde die Art der Nachbehandlung, Mobilität, Alkohol- und Nikotinmissbrauch sowie die Zeit der Immobilisation dokumentiert und ausgewertet.

Alle Patienten wurden im Jahre 2010 erneut telefonisch oder per Post kontaktiert und zu Zufriedenheit und Outcome befragt. Stellte sich bei den befragten Patienten im Verlauf eine störende Pseudoarthrose ein, wurde eine sekundäre Frakturversorgung angeboten. In den Nachuntersuchungen wurde anhand der Röntgenbilder bei den dislozierten Frakturen

- die Überlappung,

- die Translation und

- die Dislokation der Frakturenden ausgemessen.

Hinsichtlich der Risikofaktoren, die eine Pseudarthrose begünstigen, wurden alle Daten mittels multivariater Analyse statistisch ausgewertet und ein prädiktives Modell abgeleitet.

\section{Ergebnisse}

$\nabla$

Zusammenfassend kam es bei den $941 \mathrm{~Pa}-$ tienten in 125 Fällen zu einer Pseudarthrose. Prozentual entspricht dies einer Quote von 13,3\%. Geschlecht, Rauchgewohnheiten sowie der Überlappungsgrad der Frakturenden, eine Zertrümmerung und die Art der Dislokation der Fraktur waren assoziiert mit dem steigenden Risiko eine Pseudarthrose zu erleiden. Je grö- ßer die gemessene Dislokation bei gleichzeitigem Nikotinabusus war, umso signifikant höher lag die Pseudarthrosenrate der Fraktur.

\section{Kommentar}

$\nabla$

Insgesamt wurde bestätigt, dass es bei stärkerer Frakturdislokation zu einer signifikant höheren Pseudoarthroserate kommt. Nikotin hat dabei generell einen ungünstigen Einfluss auf die Knochenheilung. Dies wurde in der Vergangenheit in zahlreichen anderen Arbeiten ebenfalls bereits bestätigt. Die Chance einer vernünftigen Heilung bei fehlender Kontaktfläche ist offensichtlich nicht gegeben. Im Klinikalltag finden sich dazu häufig derartige Beispiele.

Klavikulaschaftfrakturen treten vor allem bei jungen und sportlichen Patienten auf, die bei der Ausübung einer Trendsportart auf die Schulter fallen. Das Hauptproblem dieser Betroffenen ist eher das störende äußere Erscheinungsbild, das sich durch die vorstehenden Klavikulaenden ergibt. Viele fürchten zudem die Gefahr einer Hautperforation. Leider blieben diese Aspekte in der Studie unberücksichtigt. Die vorliegenden Daten können jedoch bei der präoperativen Beratung des individuellen Patienten und der Entscheidungsfindung zur OP hilfreich sein.

In unserer Klinik wird bei der dislozierten Klavikulaschaftfraktur des jungen sportlichen Patienten eine primär operative Versorgung empfohlen. Aus der praktischen Anwendung kann im Vergleich zum primär konservativen Vorgehen mit Verfahrenswechsel, eine geringere Pseudarthroserate und bessere Funktionalität im Schulterbereich bestätigt werden.. Der Nachteil der operativ versorgten Frakturen ist bisweilen ein tastbares und störendes Implantat, etwa beim Tragen eines Rucksacks, was nach Ausheilung eine Implantatentfernung erforderlich macht.

\section{Manuela Brunk Universitätsmedizin Rostock Chirurgische Klinik und Poliklinik Abt. für Unfall-, Hand- und Wiederherstel- lungschirurgie Manuela.brunk@med.uni-rostock.de}

